[0212-7199 (2007) 24: 1; pp 35-37] ANALES DE MEDICINA INTERNA Copyright $(2007$ ARAN EDICIONES, S.L.

AN. MED. INTERNA (Madrid) Vol. 24, N. $^{\circ} 1$, pp. $35-37,2007$

\title{
Pericarditis purulenta y empiema pleural por Streptococcus pneumoniae
}

\author{
B. SUBERVIOLA CAÑAS, J. C. RODRÍGUEZ BORREGAN, A. GONZÁLEZ \\ CASTRO, E. MIÑAMBRES, F. J. BURÓN MEDIAVILLA
}

Servicio de Medicina Intensiva. Hospital Universitario Marques de Valdecilla. Santander

PURULENT PERICARDITIS AND PLEURAL EMPYEMA DUE TO

STREPTOCOCCUS PNEUMONIAE

\begin{abstract}
RESUMEN
Presentamos el caso clínico de una mujer de 59 años de edad sin historia clínica previa que ingresó en nuestra Unidad de Cuidados Intensivos (UCI) al presentar un cuadro de insuficiencia respiratoria e inestabilidad hemodinámica. Se le diagnosticó una pericarditis purulenta causada por Streptococcus pneumoniae complicada con un derrame pericárdico masivo y un empiema pleural. Se realizó una punción pericardica que no fue efectiva y el derrame pericárdico se evacuó finalmente mediante drenaje quirúrgico. El empiema pleural fue igualmente evacuado. Recibió tratamiento antibiótico y se recuperó, normalizandose la función cardia-
\end{abstract} ca.

PALABRAS CLAVE: Pericarditis purulenta. Derrame pericárdico. Empiema. Streptococcus pneumoniae.

\begin{abstract}
We present the case of a 59 years old woman with no previous clinical history of disease who was admitted in our ICU due to respiratory failure and hemodynamic inestabillity. She was found to have purulent pericarditis due to Streptococcus pneumoniae, complicated by masive pericardial effusion and pleural empyema. A pericardial tap was performed unsuccessfully and finally pericardial effusion was evacuated by surgical drainage. Pleural empyema was also evacuated. She received antibiotical treatment and recovered with normalisation of heart function.
\end{abstract}

KEY WORDS: Purulent pericarditis. Pericardial effusion. Empyema. Streptococcus pneumoniae.

Suberviola Cañas B, Rodríguez Borregán JC, González Castro A, Miñambres E, Burón Mediavilla FJ. Pericarditis purulenta y empiema pleural por Streptococcus pneumoniae. An Med Interna (Madrid) 2007; 24: 35-37.

\section{INTRODUCCIÓN}

La pericarditis purulenta, y más concretamente la causada a infección por Streptococcus pneumoniae, es una patología rara en la actualidad. La introducción de la penicilina ha supuesto un descenso drástico en su incidencia. Se trata, sin embargo, de un proceso infeccioso que evoluciona de forma inexorable hacia el fallecimiento del paciente si no es tratado de forma adecuada y aun así presenta asociadas complicaciones de gravedad. Por este motivo, la realización de un diagnóstico lo más precoz posible es fundamental. Este se encuentra fundamentado básicamente en la ecocardiografía que tiene utilidad tanto diagnostica como terapéutica, permitiendo objetivar la existencia de derrame pericárdico asociado y facilitando su evacuación mediante pericardiocentesis en aquellos casos en que exista indicación. Presentamos un caso de pericarditis neumocócica con taponamiento cardiaco cuya evolución se vio complicada por el desarrollo de varios empiemas pleurales. El diagnóstico y la evacuación precoces tanto de la colección pericárdica como de las pulmonares jugaron, en nuestra opinión, un papel crucial en la evolución satisfactoria del mismo

\section{CASO APORTADO}

Mujer de 59 años de edad sin antecedentes médicos de interés. Presentaba un cuadro de 72 horas de evolución consistente en una intensa astenia, anorexia y deterioro del nivel de conciencia que se acompañaba en las ultimas 24 horas de disnea. Acudió al Servicio de Urgencias de nuestro centro donde se objetivó insuficiencia respiratoria parcial y una fibrilación auricular con respuesta ventricular rápida e inestabilidad hemodinámica secundaria motivo por el que se decidió el traslado a la Unidad de Cuidados Intensivos (UCI).

Trabajo aceptado: 27 de julio de 2006

Correspondencia: Borja Suberviola Cañas. Servicio de Medicina Intensiva. Hospital Universitario Marqués de Valdecilla. Avda. Valdecilla, s/n. 39008 Santander. e-mail: bsuberviola@yahoo.es 
A su llegada a la UCI la paciente se encontraba obnubilada, hipotensa (tensión arterial: 90/60 $\mathrm{mmHg}$ ), en fibrilación auricular a 130 lpm, taquipnéica a $30 \mathrm{rpm}$ y con saturación arterial de oxigeno de $90 \%$ según pulsioximetría respirando con mascarilla tipo ventimask al 50\% de fracción inspirada de $\mathrm{O}_{2}$. En la exploración física destacó únicamente la existencia de ingurgitación yugular bilateral e hipoventilación en la base pulmonar izquierda. A nivel analítico llamó la atención la existencia de leucocitosis con 15.300 leucocitos, neutrofilia y una importante desviación izquierda. Electrocardiograma con fibrilación auricular rápida sin alteraciones de la repolarización. Radiografía de tórax con cardiomegalia y borramiento de ambos hemidiafragmas. Se canalizó un catéter venoso central y un catéter PICCO (5F Pulsiocath, Pulsion Medical Systems AG, Germany), objetivándose un patrón hemodinámico compatible con sepsis (gasto cardiaco elevado con resistencias vasculares sistémicas disminuidas) y unos valores de presión venosa central elevados. Recibió tratamiento con fluidoterapia, inotrópicos, amiodarona y oxigenoterapia. Se realizó un ecocardiograma transtorácico que mostró un gran derrame pericárdico con compromiso hemodinámico (Fig. 1). Se intentó una pericardiocentesis guiada por ecocardiografía obteniéndose material purulento que debido a su consistencia no permitió un drenaje efectivo, llevándose a cabo un drenaje quirúrgico con evacuación de $450 \mathrm{cc}$ de material de aspecto mucoide y coloración verdosa en que se observaron cocos gram positivos en tinción de Gram. Recibió tratamiento antibiótico empírico con meropenem y teicoplanina en espera de los resultados microbiológicos definitivos. Se aisló Streptococcus pneumoniae sensible a penicilina decidiéndose tratamiento con penicilina $\mathrm{G}$ sódica endovenosa en monoterapia.

Presentó un incremento del trabajo respiratorio y un empeoramiento de los parámetros gasométricos por lo que se procedió a su intubación y conexión a ventilación mecánica (VM). Transcurridas 72 horas del ingreso en la UCI, se realizó una nueva TAC torácica observandose en el hemitorax izquierdo un derrame pleural multitabicado (Fig. 2). Se realizó una toracocentesis guiada por ecografía obteniéndose liquido turbio con $\mathrm{pH} 7,10$. Se colocó un tubo de drenaje torácico y se inició instilación de urokinasa a dosis de 100.000 unidades cada 8 horas por el mismo. Tras 3 días de tratamiento se obtuvieron $600 \mathrm{cc}$ de líquido de aspecto purulento, realizándose un nuevo TAC torácico que permitió confirmar la desaparición prácticamente total de los empiemas. En ecocardiogramas sucesivos se mantuvo un mínimo derrame pericárdico, sin signos de taponamiento y con imágenes de organización en su interior. La evolución posterior fue satisfactoria a excepción de un destete de la VM dificultoso que requirió realización de traqueostomía percutánea al día $15^{\circ}$ de ingreso, siendo finalmente dada de alta.

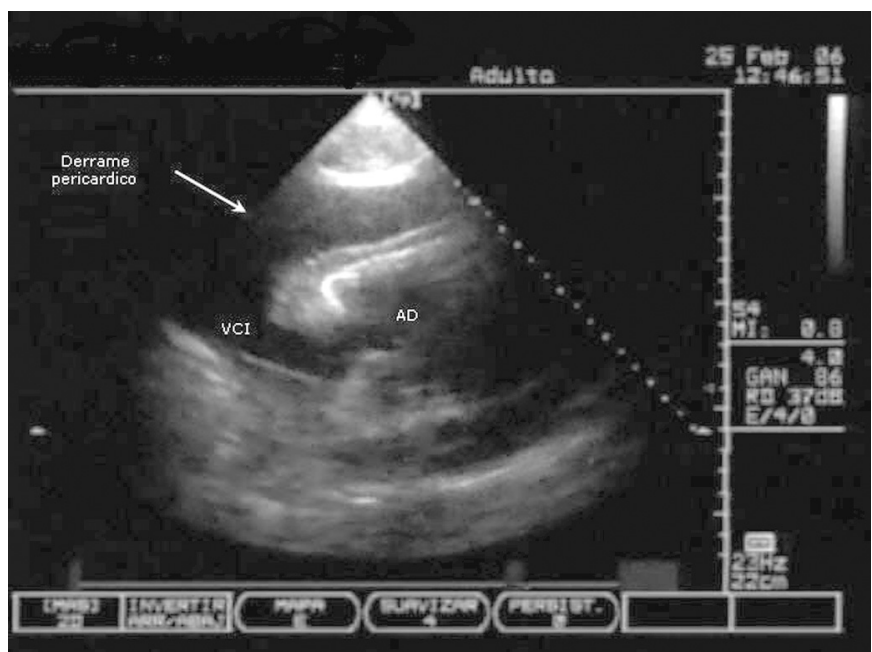

Fig. 1.

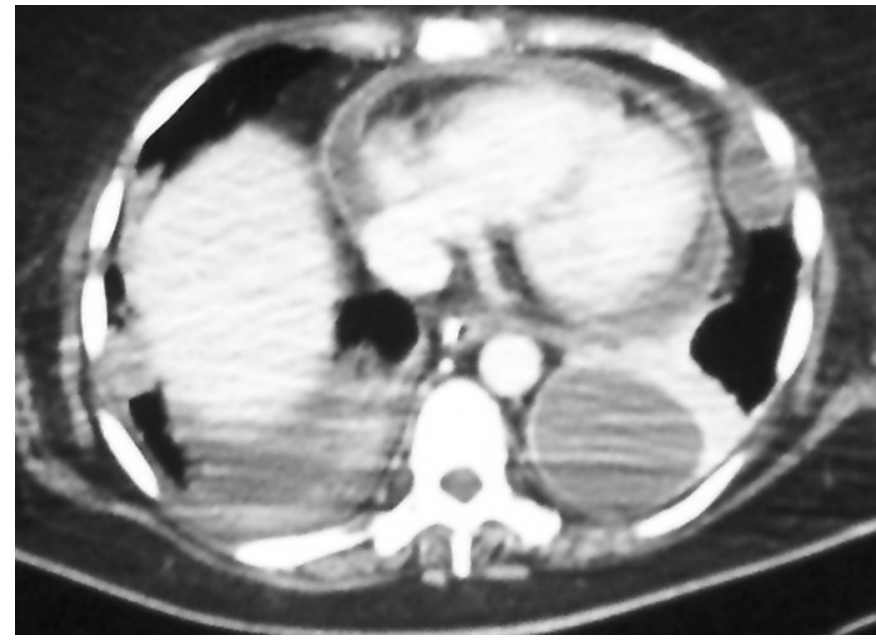

Fig. 2.

\section{DISCUSIÓN}

Aunque se han descrito numerosas causas de pericarditis aguda, en nuestro medio la etiología más frecuente es la idiopática o viral, especialmente en pacientes ambulatorios $(1,2)$. Este dato sería aplicable igualmente a los taponamientos cardiacos. Así, y aunque en los últimos años estamos asistiendo a un incremento de las etiologías especificas (fundamentalmente neoplásicas), la pericarditis aguda idiopática continúa siendo la causante de la mayor parte de los taponamientos cardiacos. Los derrames pericárdicos asociados tanto a la pericarditis purulenta como a la tuberculosa presentan una baja incidencia en nuestro país.

En un estudio realizado sobre 96 pacientes que presentaban taponamiento cardiaco (3) se objetivo origen neoplásico en el $52 \%$ de los casos, idiopático en el $14 \%$ y solo en uno de los casos ( $1 \%$ del total) fue una pericarditis purulenta la responsable del taponamiento cardiaco. En otra serie de 204 pacientes, solo en 107 casos (52\%) se logro el diagnóstico etiológico, con tan solo 19 casos atribuibles a infección bacteriana no tuberculosa (4).

La pericarditis purulenta en individuos adultos es rara, pero su evolución es fatal si no recibe un tratamiento antibiótico adecuado. Ocurre más frecuentemente como consecuencia de la migración de gérmenes al saco pericárdico provenientes de un foco séptico preexistente. Los mecanismos para su desarrollo son múltiples: extensión directa desde un foco infeccioso intratorácico, foco miocárdico, infección directa por trauma o cirugía torácica, extensión desde un foco infeccioso subdiafragmático o por diseminación hematógena. La infección primaria es muy rara (5). Los organismos más comúnmente implicados son Staphylococcus aureus, Haemophilus influenzae, Neisseria meningitidis y Streptococcus pneumoniae; menos frecuentemente se aíslan bacilos gramnegativos entéricos, Pseudomona aeruginosa, Salmonella, anaerobios y hongos.

La pericarditis purulenta se suele presentar como complicación de un proceso infeccioso ubicado en cualquier otro punto del organismo. Son factores predisponentes para su presentación la existencia previa de derrame pericárdico, la uremia, inmunosupresión, diabetes, enfermedades crónicas (alcoholismo, artritis reumatoide, etc.), cirugía cardiaca y el 
traumatismo torácico. En el caso concreto de la pericarditis purulenta de origen neumocócico, la afectación pericárdica se produce más frecuentemente por contigüidad a partir de un foco neumónico y en menor proporción por diseminación vía hematógena (6). La enfermedad se presenta de forma aguda, como un proceso infeccioso fulminante en el que predominan una gran afectación general, con fiebre, taquicardia y taquipnea sobre los síntomas clásicos de pericarditis. La existencia de dolor torácico es poco frecuente.

El diagnóstico se sustenta sobre dos pilares fundamentales, la clínica y el ecocardiograma. El ecocardiograma es la exploración más útil para identificar la presencia y cuantificar el derrame pericárdico. Puede aportar además, datos muy útiles para establecer él diagnóstico de taponamiento, fundamentalmente colapso de la aurícula o del ventrículo derechos y alteraciones de los flujos mitral y tricuspídeo (7). Junto a su utilidad diagnóstica, permite ayudar a la realización de pericardiocentesis completando de esta forma el diagnóstico etiológico. Es una técnica segura especialmente si se practica por personal adecuadamente entrenado. Recientemente se ha descrito la utilidad de la TAC como método de diagnóstico precoz de la existencia de derrame pericárdico. Se trata de un método no invasivo, que permite no sólo cuantificar la magnitud del derrame sino realizar una aproximación diagnóstica a la etiología del mismo (8).

El tratamiento adecuado requiere antibioterapia intravenosa, drenaje pericárdico y medidas de tratamiento intensivo. El tratamiento antibiótico inicial debe cubrir Staphylococcus aureus y bacilos gramnegativos, recomendándose una combinación de un betalactámico resistente a penicilinasa con una cefalosporina de $3^{\mathrm{a}}$ generación, considerando el uso de vancomicina en áreas de elevada tasa de resistencia estafilocócica. Se recomienda la asociación de un aminoglucósido en caso de inmunosupresión, en el postoperatorio de cirugía cardiaca o cuando el foco primario es genitourinario. La antibioterapia intrapericárdica no parece tener especial indicación dada la excelente penetración de los antimicrobianos en la cavidad pericárdica. El rendimiento de los cultivos de liquido pericár- dico en los pacientes con pericarditis purulenta es elevado, con una tasa de determinaciones positivas que oscila entre el 50 y $60 \%$. La duración del tratamiento es empírica, pero habitualmente se debe prolongar al menos durante 3 ó 4 semanas (9).

La realización de una pericardiocentesis evacuadora es obligada en aquellos casos que cursen con inestabilidad hemodinámica, y esta indicada así mismo en colecciones de más de $20 \mathrm{~mm}^{3}$ cuantificadas por ecocardiografía (en diástole). La coexistencia de disección aórtica se considera una contraindicación mayor para la realización de la técnica mientras que entre las relativas destacan la existencia de una coagulopatía no corregida, tratamiento anticoagulante, trombocitopenia $<50.000$ plaquetas $/ \mathrm{mm}^{3}$, colecciones pericárdicas pequeñas, posteriores y loculadas. El mantenimiento del drenaje pericárdico debe prolongarse hasta que la cantidad de liquido obtenido por drenaje aspirativo intermitente (cada 4 a 6 horas) sea inferior a $25 \mathrm{ml} /$ día. El drenaje quirúrgico es preferido en casos de hemopericardio traumático y en pericarditis purulenta (10).

La mortalidad del cuadro oscila entre el 2 y $20 \%$ en aquellos casos que reciben tratamiento adecuado. Son factores de mal pronóstico el retraso en el drenaje pericárdico, la existencia de taponamiento en el momento del diagnóstico, la infección por Staphylococcus aureus o bacilos gramnegativos y la malnutrición. La secuela mas frecuente, aunque inusual, es la pericarditis constrictiva que ocurre generalmente en las semanas siguientes al diagnóstico. Otras complicaciones menos frecuentes son la formación de aneurismas micóticos aórticos, la infección miocárdica y la bacteriemia sistémica (9).

El caso clínico que presentamos es un ejemplo de la complejidad y gravedad de esta patología, que aunque poco frecuente, presenta una alta tasa de mortalidad asociada. La sospecha de esta entidad justifica, en nuestra opinión, la utilización de métodos de diagnóstico precoz y su manejo agresivo con el fin de evitar no sólo el fallecimiento del paciente sino también el desarrollo de complicaciones, que cuentan con una importante morbi-mortalidad.

\section{Bibliografía}

1. Sagrista Sauleda J. Pericarditis aguda. Med Clin (Barc) 2004; 123: 5058

2. Sagrista Sauleda J, Permanyer Miralda G, Soler Soler J. Diagnosis and management of acute pericardial syndromes. Rev Esp Cardiol 2005; 58: $830-41$.

3. Oliver Navarrete C, Marín Ortuno F, Pineda Rocamora J, Lujan Martínez J, García Fernández A, Climent Payá VE, et al. Should we try to determine the specific cause of cardiac tamponade? Rev Esp Cardiol 2002; 55: 493-8.

4. Levy PY, Corey R, Berger P, Habib G, Bonnet JL, Levy S, et al. Etiologic diagnosis of 204 pericardial effusions. Medicine 2003; 82: 385-91.

5. Álvarez Navascues R, Hsieh Ching CJ, Moller I, Quinones Ortiz L, Guerediaga Madariaga J. Purulent pericarditis caused by Streptococcus agalactiae. An Med Interna (Madrid) 2005; 22: 198.

6. Hastbacka J, Kolho E, Pettila V. Purulent pneumococcal pericarditis: a rarity in the antibiotic era. J Crit Care 2002; 17: 251-4.

7. Spodick DH. Acute pericarditis: current concepts and practice. JAMA 2003; 289: 1150-3.

8. Rifkin RD, Mernoff DB. Noninvasive evaluation of pericardial effusion composition by computed tomography. Am Heart J 2005; 149: 1120-7.

9. Albalá Martínez N, Moneo González A, Waez Tatari B, Argüelles Baquero A, Ferrero Zorita J, Martín Benítez JC. Pericarditis neumocócica: presentación de un caso y revisión de la literatura. Med Intensiva 2005; 29: 308-12.

10. Maisch B, Seferovic PM, Ristic AD, Erbel R, Rienmuller R, Adler Y, Tomkowski WZ, Thiene G, Yacoub MH. Guidelines on the diagnosis and management of pericardial diseases executive summary; The Task force on the diagnosis and management of pericardial diseases of the European society of cardiology. Eur Heart J 2004; 25: 587-610. 